\title{
USING THE PROGRAMMING LANGUAGE IN STUDYING THE DISTRIBUTION NORMAL RANDOM QUANTITIES FUNCTION
}

\section{Ruzimurodov Ikhtiyor Nishonovich}

Asistant of Karshi branch of TUIT

\begin{abstract}
:
This article uses $\mathrm{C}++$ programming to plot the distribution function of a normal random variable. In addition, it was noted that interdisciplinary integration plays an important role in preparing future programmers for professional activities.

Keywords: Random quantity, distribution function, parameter, software tools, program code, function graph, integration, compilation.
\end{abstract}

In order to increase the effectiveness of the educational process and further improve it, it is important to choose and use the most scientifically based forms, methods and tools of education. One of the most important tools is to organize the educational process in an interdisciplinary way.

The creation of problem-solving problems in the organization of the practical training process and the use of software to solve them serve the following didactic purposes:

The student focuses on solving the problem, stimulates the atmosphere of knowledge and performance, as well as other aspects of the activity.

During the practical training, the student overcomes some of the complexities and difficulties encountered in the use of software to solve problems, as well as develops independent thinking and learning activities.

Eliminates the discrepancy between the demand for knowledge that arises during the use of software to solve problems in the course of practical training for the student and the inability to meet this demand through existing theoretical knowledge, practical skills and abilities.

Defines a plan for the student to find ways to get rid of the difficulties and encourages him / her to actively seek [4].

In view of the above, we will mention the use of software tools, mainly programming languages, in the study and graphing of normal distribution functions.

A general normal distribution is an arbitrary and () parametric normal distribution.

$$
F(x)=\frac{1}{\sigma \sqrt{2 \pi}} \int_{-\infty}^{x} e^{-\frac{(z-a)^{2}}{2 \sigma^{2}}} d z
$$

11.appears in [1].

The standard is called the normal distribution and the parameter is called the normal distribution.

The density function of the standard normal distribution

It's easy to see that it's in place.

The probability that an arbitrary and parametric normal random quantity takes a value corresponding to the interval can be found using the Laplace function. Indeed, we'll see

We'll add a new variable. From here, it follows that. We find new frontiers of integration. If so, so be it; if so, then so be it.

And SO,

$$
\phi(x)=\frac{1}{\sqrt{2 \pi}} e^{-\frac{x^{2}}{2}}
$$

using the function, eventually 


$$
\text { 13. } \Phi(x)=\frac{1}{\sqrt{2 \pi}} \int_{0}^{x} e^{-\frac{z^{2}}{2}} d z
$$

we get

In particular, the probability that the standard $\mathrm{X}$ takes a value corresponding to the range of a normal random quantity

$$
\begin{gathered}
14 P(\alpha<X<\beta)=\int_{\alpha}^{\beta} f(x) d x=\frac{1}{\sigma \sqrt{2 \pi}} \int_{\alpha}^{\beta} e^{-\frac{(x-a)^{2}}{2 \sigma^{2}}} d x \\
P(\alpha<X<\beta)=\frac{1}{\sigma \sqrt{2 \pi}} \int_{(\alpha-a) / \sigma}^{(\beta-a) / \sigma} e^{-\frac{z^{2}}{2}}(\sigma d z)=\frac{1}{\sqrt{2 \pi}} \int_{(\alpha-a) / \sigma}^{0} e^{-\frac{z^{2}}{2}} d z+\frac{1}{\sqrt{2 \pi}} \int_{0}^{(\beta-a) / \sigma} e^{-\frac{z^{2}}{2}} d z= \\
=\frac{1}{\sqrt{2 \pi}} \int_{0}^{(\beta-a) / \sigma} e^{-\frac{z^{2}}{2}} d z-\frac{1}{\sqrt{2 \pi}} \int_{0}^{(\alpha-a) / \sigma} e^{-\frac{z^{2}}{2}} d z .
\end{gathered}
$$

is equal to, because in this case and [5].

The mathematical expectation of a normally distributed random variable $\mathrm{X}$ is equal to the standard deviation of $\mathrm{a}=3$. Suppose you want to write a differential function of $\mathrm{X}$.

$$
\begin{gathered}
P(\alpha<X<\beta)=\Phi\left(\frac{\beta-a}{\sigma}\right)-\Phi\left(\frac{\alpha-a}{\sigma}\right) \\
f(x)=\frac{1}{2 \sqrt{2 \pi}} e^{-\frac{(x-3)^{2}}{8}}
\end{gathered}
$$

In Microsoft Excel, the function graph is created as follows:

\begin{tabular}{|l|l|}
\hline $\mathbf{X}$ & $\mathbf{y = f ( x )}$ \\
\hline-4 & 0,00274161 \\
\hline-3 & 0,01392306 \\
\hline-2 & 0.0550667 \\
\hline-1 & 0,16961762 \\
\hline 0 & 0,40689152 \\
\hline 1 & 0,76017341 \\
\hline 2 & 1,10604584 \\
\hline 3 & 1,25331413 \\
\hline 4 & 1,10604584 \\
\hline 5 & 0,760173451 \\
\hline 6 & 0,406891527 \\
\hline 7 & 0,169617624 \\
\hline 8 & 0,05506678 \\
\hline
\end{tabular}

In the $\mathrm{C}++$ programming language, we create a graph of the distribution function as follows:

We run the Borland $\mathrm{C}++$ builder programming language;
We place the following components from the component palette on the form:

From the System component, we place the PaintBox component on the form; 
From the standard content, we place the Button component on the form;

We denote the button by the name OK [2].

Double-click the left mouse button on the OK button and enter the following program code:

\#include <vcl.h>

\#include <math.h>

\#pragma hdrstop

\#include "Unit1.h"

$/ /$

\#pragma package(smart_init)

\#pragma resource "*.dfm"

TForm1 *Form1;

$/ /$

Owner)

void_fastcall TForm1::Button1Click(TObject *Sender)

double $\mathrm{g}=10, \mathrm{t}, \mathrm{v} 0, \mathrm{pi}=3.1415926$;

double $\mathrm{x}, \mathrm{x} 1, \mathrm{f}, \mathrm{h}, \mathrm{x} 2, \mathrm{x} 0, \mathrm{y} 0$, fx,fy;

Canvas->MoveTo(450,200);

Canvas->LineTo(440,205);
Canvas->MoveTo(450,200);

Canvas->LineTo(440,195);

Canvas->MoveTo $(10,0)$;

Canvas->LineTo(5,15);

Canvas->MoveTo $(10,0)$;

Canvas->LineTo(15,15);

Canvas->MoveTo(10,200);

Canvas- $>\operatorname{LineTo}(10,0)$;

Canvas->MoveTo(450,200);

Canvas->LineTo(10,200);

Canvas->TextOutA(20,5,"F(x)");

Canvas->TextOutA(450,210,"x");

$\mathrm{x} 0=0 ; \mathrm{y} 0=200$;

$\mathrm{x} 1=0 ; \mathrm{x} 2=400$;

$\mathrm{x}=\mathrm{x} 1$;

while $(x<x 2)$

\{

$\mathrm{fx}=\mathrm{x} 0+10 * \mathrm{x}$

fy $=\mathrm{y} 0-500 *(1 /(2 * \operatorname{sqrt}(2 * \mathrm{pi}))) * \exp \left(0.05^{*}(-(\mathrm{x}-\right.$

$\left.\left.3)^{*}(\mathrm{x}-3) / 8\right)\right)$;

PaintBox1->Canvas->Pixels[fx][fy]=clBlue; $\mathrm{x}=\mathrm{x}+0.0005$

When you click OK during the compilation process, the following graphic image will appear [2],

[3]:

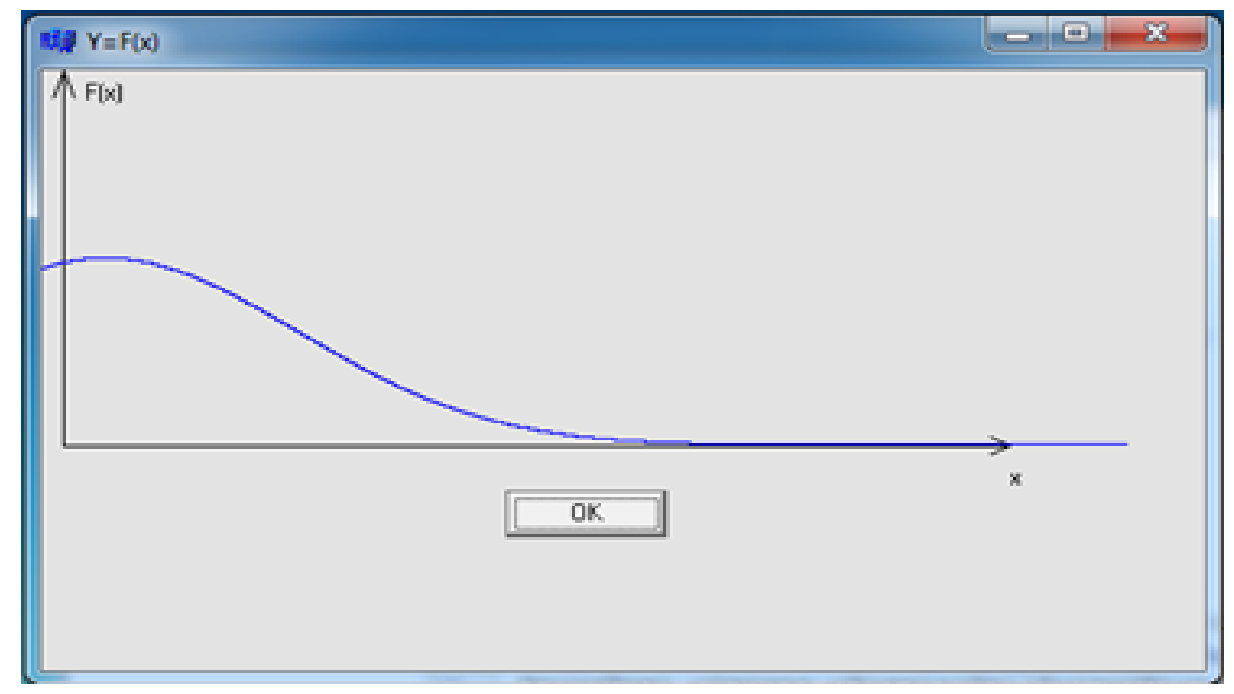

As can be seen from the graph, the distribution function is continuous with increasing and decreasing intervals. The maximum value of the distribution function is determined by calculating the first-order product and setting it to zero.

The $\mathrm{C}++$ programming language is inextricably linked to the science of probability theory, in which it is important to express, implement, and experiment with any possible 
event through program code. At the same time, the emphasis on interdisciplinary links in improving the professional preparation of future programmers is an important factor in improving the quality of education [4].

Practical issues play an important role in achieving interdisciplinary connections in the teaching of probability theory and mathematical statistics, and they help students first understand probability theory and mathematical statistics as a science. Second, solving practical problems leads to students acquiring the skills to apply mathematical knowledge directly in the study of other disciplines and to understand the knowledge of the subject.

\section{References}

[1] Soatov Yo. Higher mathematics. Tashkent "Teacher", 1995.

[2] Horstsman, Gay S. C++ for everyone/Gay S. Horstsman. Printed in the United States of America-2nd ed. 2010.-P.562.

[3] Horton I.-Beginning Visual C++ 2012/ I. Horton. Published imultaneously in Canada.-2012.-P.988.

[4] Turaev S.J. Methods of the using of software program Microsoft Excel in practical and laboratory occupation on Physics. Scientific Bulliten on Namangan State University: Vol.1: Iss.10, Article 55. -Namangan.:2019. -P. -292-297.

[5] Gmurman V.E. Probability theory and mathematical statistics: A textbook for universities. M.: 2003, -p.479

[6] Danko P.S., Popov A.G., Kozhevnikova T.Ya. Higher mathematics in exercises and problems. At 2 o'clock -M .: Higher; school, 1998.-h. 12.

[7] Sirojiddinov S.X., Mamatov M. Probability Theory Course. T. Teacher, 1980.

[8] Minorsky V.I. Collection of problems in higher mathematics. M: Science, 1987.

[9] Jo'raev T., Sa'dullaev A., Khudoiberganov G., Mansurov X., Vorisov A.
Fundamentals of Higher Mathematics. T.1., Tashkent, “Teacher", 1995.

[10] Jo'raev T., Sa'dullaev A., Khudoiberganov

G., Mansurov X., Vorisov A. Fundamentals of Higher Mathematics. T.2., Tashkent, “Uzbekistan”, 1999. 